\title{
Spontaneous imbibition in asymmetric branch-like throat structures in unconventional reservoirs
}

\author{
Yinghao Shen a , Caoxiong Li a, b, c, *, Hongkui Ge a, Xinyu Yang a, d, Xuxin Zeng e \\ a Unconventional Natural Gas Institute, China University of Petroleum, Beijing 102249, China \\ ${ }^{\mathrm{b}}$ Institute of Mechanics, Chinese Academy of Sciences, Beijing 100190, China \\ c School of Engineering Science, University of Chinese Academy of Sciences, Beijing, 100049, China \\ d CNPC Bohai Drilling Engineering Company Limited, Tianjin, 300457, China \\ e College of Petroleum Engineering, China University of Petroleum (East China), Qingdao, Shandong, 266580, China
}

\section{A R T I C L E I N F O}

\section{Article history:}

Received 11 October 2016

Received in revised form

23 January 2017

Accepted 23 April 2017

Available online 26 April 2017

\section{Keywords:}

Unconventional reservoir

Spontaneous imbibition

Fractals

\begin{abstract}
A B S T R A C T
Unconventional reservoirs, especially shale plays, have abundant micro-nano pores. Large volume water was injected into the reservoir during hydraulic fracturing. Fracturing fluid can displace gas in pore spaces spontaneously, called spontaneous imbibition (SI), which has significant effects on the gas production after hydraulic fracturing. Throat structure in matrix is one of the key factors to influence SI process. In this work, at first, complex pore space is simplified as a spatial self-similar branch-like structure composed of several asymmetric sub-throats. Then, within asymmetric branch structure, the imbibition process is calculated by a newly established mathematical model, and the influence of both pore structure and fluid characteristics, including contract angle, viscosity, surface tension, are discussed. Finally, some lab experiments, especially SI tests, are included on shale and sandstone core plugs to verify our model. Results show that the coherence in wettability between the reservoir and the fracturing fluid is important to enhance the microscopic replacement effect. Pore size also has effect influence on imbibition curve. In addition, both in models and experiments, imbibition index $\mathrm{n}$ is useful to describe imbibition properties of rocks. In unconventional rock samples like shale, $\mathrm{n}$ is less than 0.4 , while highpermeability sandstone, $\mathrm{n}$ approach to 0.5 . These conclusions are useful to investigate the mechanism of spontaneous imbibition and microscopic displacement in unconventional reservoirs.
\end{abstract}

() 2017 Elsevier B.V. All rights reserved.

\section{Introduction}

The increasing demand for energy has prompted the need to find economical ways of developing unconventional resources, such as shale oil and gas. Compared with conventional reservoirs, a shale gas reservoir has relatively low permeability and porosity most hydrocarbon is stored in its tight matrix. Shale gas reservoirs require volume fracturing technologies, during this process, tons of fracturing liquids are pumped in reservoir. Blocked fracturing liquid in pore structures is critical for gas production (Yuan et al, 2015a, 2015b; Moghanloo et al., 2015). The ability of liquid imbibition, liquid diffusing and self-unblocking is critical to the successful development of shale gas reservoirs (Shen et al., 2016).

\footnotetext{
* Corresponding author. Unconventional Natural Gas Institute, China University of Petroleum, Beijing 102249, China.
}

E-mail address: licaoxiong@imech.ac.cn (C. Li).
Spontaneous imbibition (SI), which is caused by capillary forces, is one of control factors to the liquid uptaking and diffusing ability. Qasem et al. (2008) determined that co-current and countercurrent imbibition have microscopic displacement functions throughout the imbibition process. Roychaudhuri et al. (2011) pointed out that, on the basis of shale gas reservoir development practices, a shale gas well has a high yield at low flowback rate; this phenomenon is closely related to SI in reservoirs. Bertoncello et al. (2014) found that SI is strongly related to fluid loss in reservoirs.

In tight matrix blocks, complex pore space is one of the important factors that influence SI laws. The matrix pore network is the main passage for fracturing fluid. Controlled by capillary force, the fracturing fluid penetrates the matrix through tortuous, complex pore spaces. Rose (2001) and Clarkson et al. (2011) claimed that this phenomenon involves a complex interaction among viscosity, surface tension, contact angle, capillary force, gravity, wettability, porosity, and pore structure. Numerical simulation should be performed to identify the key factors and mechanism 
that influence SI. Unfortunately, pore spaces are too complex. Thus, a relevant model needs to be simplified. Lucas (1918) and Washburn (1921) studied the movement laws of the front edge of SI in vertical throats. Handy (1960) simplified rocks as capillary bundles and proposed the earliest SI model, which considered that the cumulative core imbibition mass was linear with the square root of imbibition time. Zhang et al. (1996), Li and Horne (2001), and Schechter et al. (1994) contributed to the development of a SI model driven by capillary force under different conditions. However, those models do not take into consideration the effects of pore tortuosity and intersection on SI, and merely simplified pore space as groups of capillary tubes.

With the use of self-similarity of complex throats, the pore space is considered to be a network system that consists of spatially asymmetric sub-structures that act as a representative elementary volume (REV). This REV shares the same SI property with the pore space. Under these simplifications, Xu et al. (2006) studied the seepage characteristics of tortuous and cross throats. Luo et al (2010) studied the flow regularity of non-Newtonian fluids in tortuous, asymmetric tree-like sub-throats. Pia (2016) and Pia et al. (2016) used Intermingled Fractal Units' model to discuss the permeability and SI characteristics of porous media.

Rock is a porous medium, and its pore structure has fractal characteristics (Mandelbrot, 1982; Katz and Thompson, 1985; Krohn and Thompson, 1986), so does fracture networks (Ankur et al., 2007). Fractal dimension can describe the tortuosity in throats. When the fractal dimension is 1 , the throat is basically straight; when it is close to 3 , the throat is sufficiently complex to fill three-dimensional spaces. By introducing fractal theory, Cai et al. (2010a) studied the SI law of single tortuous capillary. Furthermore, they developed this theory and made many applications in porous media based on fractal geometry (Cai et al., 2010b, 2012, 2014, 2015). Sang (2012) studied the SI characteristics of a symmetric tree-like structure. Fractal theory has provided an important way to describe tortuous capillary.

SI in matrix is influenced by many factors, among which the key control factors are unclear. Studying SI in reservoirs on the basis of REV imbibition laws is important. Such a study may provide suggestions on fracturing process in-situ with regard to decisions on field fracturing processes and the choice of flow back time.

\section{Basic representative elementary structure}

SI in matrix is a process in which fracturing fluid spontaneously enter spore spaces to displace hydrocarbon microscopically and to have physical and chemical interactions with clay mineral (Engelhardt and Tunn, 1955), as shown in Fig. 1. To research the effects of pore structure on the imbibition process, we ignored the clay mineral factor and focused only on tortuosity, connectivity, and branches. Therefore, pore space is simplified as a space net structure that retains pore-throat tortuosity, connectivity, and the topological structure. The imbibition process in this structure reflects the actual mechanism of reservoir imbibition. Additionally, nonNewton characteristics of fluid, low connectivity of matrix, compressibility of gas and matrix are not discussed in this model.

In a space net structure, the most basic representative elementary volume is asymmetric sub-throat structure, as shown in Fig. 1. This structure mainly includes the following parts: the main throat, where the fracturing fluid first soaks into the lower part; the branch point, where the flow channel forks; and the subthroat, which is the forked throat. A tortuous throat is not always straight. In this paper, the length of lines between start and end points of one throat is called linear length; the projection of inthroat liquid velocity in a linear length direction is called linear velocity; the actual length of the axis of single throat is called streamline length; and the velocity at which the liquid flows along the pathline's tangential direction is called actual velocity. The introduction of REV has simplified the object of study, thereby making a study of the imbibition process in a complex throat structure convenient

This simplified model calculates the imbibition process of fracturing fluids, i.e., the absorbed water mass changes over time. Hu et al. (2012) and Makhanov et al. (2014) suggested that these parameters should meet the following equation:

$M(t)=A t^{n}$

where $A$ denotes the imbibition rate, which reflects the speed of imbibition mass at the initial stage of SI and is related to pore diameter, contact angle, viscosity, and surface tension; $n$ denotes the imbibition index, which mainly reflects the pore connectivity

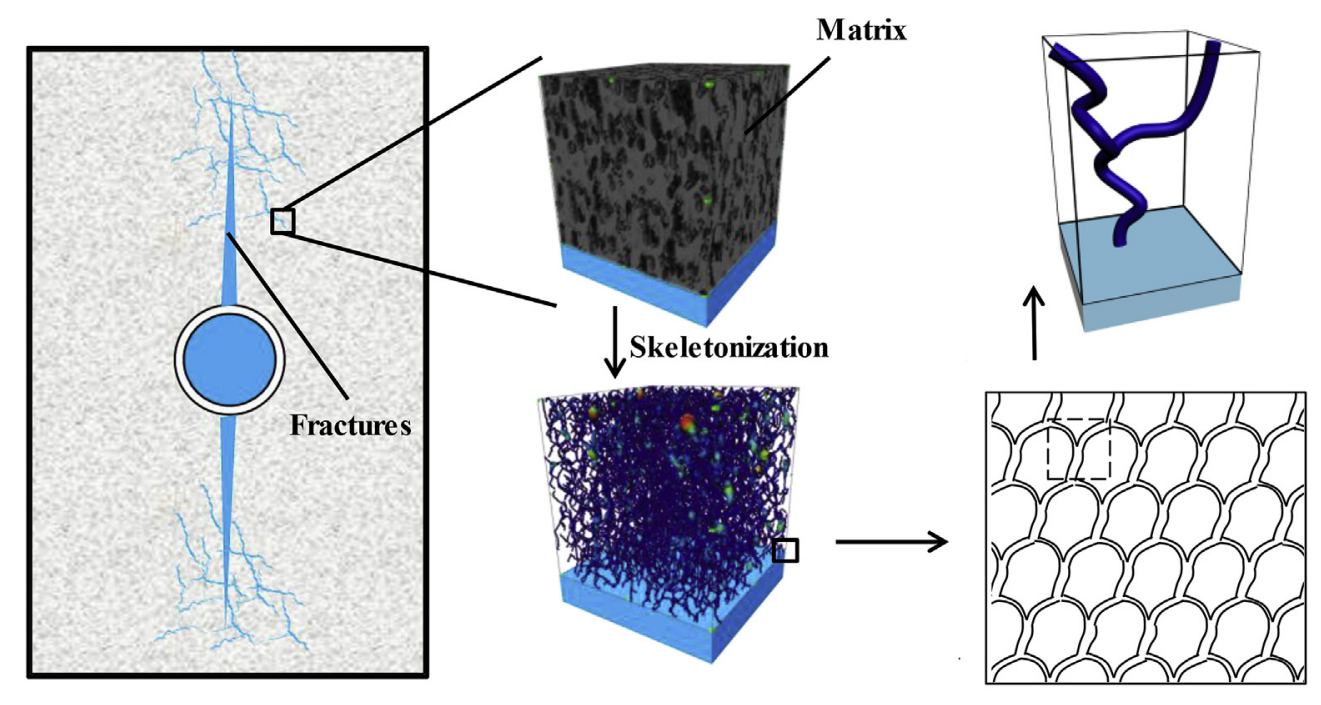

Fig. 1. Matrix imbibition versus asymmetric sub-throat structure. 
and complexity, and is related to pore tortuosity and pore size distribution; and $M$ denotes the core imbibition mass, which mainly reflects the water absorbing capacity. To directly calculate $n$ value, the above curve is sometimes described in logarithmic coordinates; at this point, the imbibition index $n$ manifests as the slope of a straight line segment on the curve in logarithmic coordinates. On the theoretical SI mechanism, Cai and Yu (2011) defined $n$ as imbibition time exponent. It is worth noting that the exponent $n$ is analytically expressed as a function of tortuosity fractal dimension, and in the range of $1 / 6-1 / 2$ in Cai and Yu's fractal model.

Imbibition process in this structure represents the process in space net structure, hence partially representing the imbibition rules of an actual pore space. On the basis of this model, we can analyze the main factors that affect SI in a rock matrix. The model simplification condition in this article is as follows: the fluid flow process is simplified as an incompressible Newtonian fluid that flows laminarly in throats regardless of wall and clay mineral effects. We mainly focus on the effects of the pore structure on the imbibition process.

\section{Modeling and solutions}

Fig. 2 shows a basic cell for a typical tree-like sub-structure. Driven by capillary force, the fracturing fluid enters the main throat and then the sub-throats via a branch point to continue the imbibition process until it flows out of the basic cell. In this process, capillary force drives liquid flowing against gravity. In isometric tubes, capillary force generally remains unchanged while gravity increases as liquid imbibed in tubes. At a certain height, the capillary force is balanced with gravity, this height is called balance height. When the branch point is higher than the balance height, this process is single-capillary spontaneous imbibition. According to the study of Lucas (1918) and Washburn (1921), this process conforms to the equation (Cai et al., 2010a):

$\frac{d L_{f}}{d t}=\frac{\lambda^{2}}{32 \mu L_{f}}\left(\frac{4 \sigma \cos \theta}{\lambda}-\rho g L_{C}\right)$

the balance height $L_{e}$ is reached as

$L_{e}=\frac{4 \sigma \cos \theta}{\lambda \rho g}$

where $L_{f}$ refers to the fluid path line length, $L_{C}$ is the vertical height of the fracturing fluid, $\lambda$ is the throat diameter, $\mu$ denotes fluid viscosity, $\sigma$ denotes fluid surface tension, $\theta$ represents wall-fluid contact angle, and $\rho$ denotes the fracturing fluid density. Given straight and vertical capillaries, $L_{f}=L_{C}$. Assuming incompressible ideal fluid flows laminar in throats, gravity is negligible at the first stage of imbibition, and Equation (1) is thus simplified as

$L_{C}=\sqrt{\frac{\lambda \sigma \cos \theta}{4 \mu}} \sqrt{t}$

Equation (3) indicates that, at the first stage of spontaneous imbibition, $L_{C}$ is proportional to the 0.5 th power of time. Eqs. (1)-(3) are Lucas-Washburn (LW) equations (Lucas, 1918; Washburn, 1921). Considering tortuous capillaries, Yu and Cheng (2002) introduces fractal dimension and then expresses the relation of $L_{f}$ and $L_{C}$ as

$L_{f}=\lambda^{1-D_{T}} L_{C}^{D_{T}}$

So the relation of actual velocity $v_{f}$ and linear velocity $v_{C}$ can be expressed as

$v_{f}=D_{T} L_{S}^{D_{T}-1} \lambda^{1-D_{T}} v_{C}$

where $D_{T}$ refers to the fractal dimension of a tortuous capillary, $\lambda$ denotes the capillary diameter. $D_{T}$ can be calculated by box counting method or by Krohn's formula (Krohn, 1988). In the following sections, $D_{T}$ is used to express the tortuosity. Cai et al. (2010a) deduced the time-dependent change of the imbibition height

$\frac{d L_{C}}{d_{t}}=\frac{\lambda^{2 D_{T}-1} \sigma \cos \theta}{8 \mu D_{T} L_{C}^{2 D_{T}-1}}-\frac{\lambda^{2 D_{T}} \rho g}{32 \mu D_{T} L_{C}^{2 D_{T}-2}}$

The pore throat factor of this model can be expressed as $L_{S} / \lambda$ in Eq. (6). When the branch point is lower than the balance height, the fluid divides into two sub-throatswith a diameter of $d_{1}$ and $d_{2}$, respectively; the contact angle against the fluid is identical, and the fluid moves up with the help of capillary force. On this basis, the capillary pressure exposed to the fluid is

$P_{C 1}=4 \sigma \cos \theta / d_{1}, P_{C 2}=4 \sigma \cos \theta / d_{2}$

The fluid flow from the main throat to the branched throat is simplified as an incompressible Newtonian fluid that flows laminarly in throats. The non-Newton characteristics of fluid are not discussed in this work. Flow in pores are considered to follow Hagen-Poiseuille law assumptions. According to Hagen-Poiseuille law and the energy conservation equation, the main throat and the

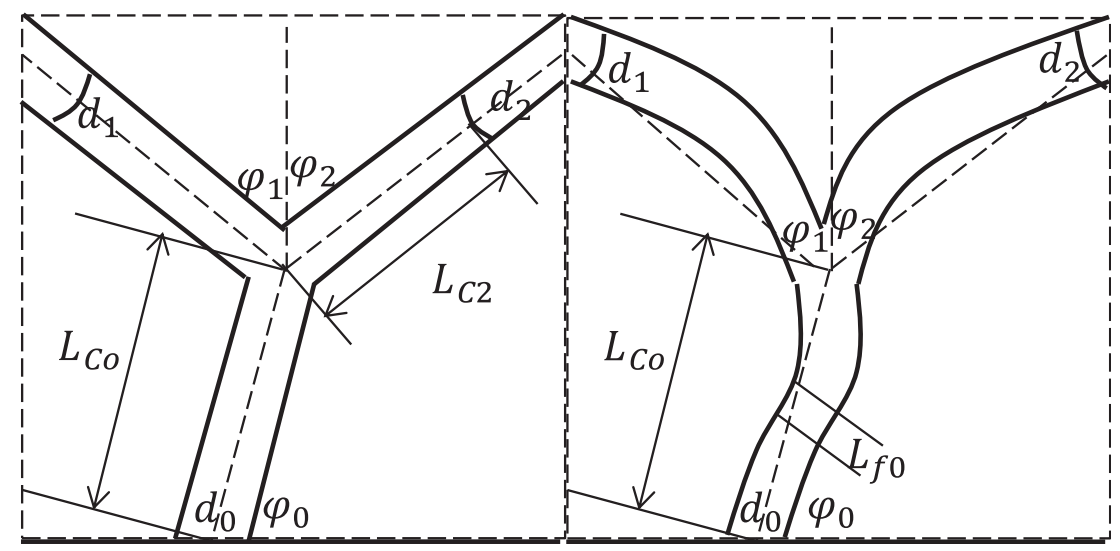

Fig. 2. Spontaneous imbibition in a typical asymmetric sub-throat. 
sub-throat should have the same water pressure when converted to the branch point, with the bottom of the main throat as the reference plane, i.e.,

$P_{C 1}-L_{C 1} \cos \varphi_{1} \rho g-\frac{32 \mu L_{f 1} v_{f 1}}{d_{1}^{2}}=P_{C 2}-L_{C 2} \cos \varphi_{2} \rho g-\frac{32 \mu L_{f 2} v_{f 2}}{d_{2}^{2}}$

$=L_{C 0} \cos \varphi_{0} \rho g+\frac{32 \mu L_{f 0} v_{f 0}}{d_{0}^{2}}$

where $L_{C 0}$ refers to the linear length of the main throat; $\phi_{0}$ refers to the angle from the vertical line; $\phi_{1}$ and $\phi_{2}$ are the angles of the subthroats, respectively; $v_{f 0}, v_{f 1}, v_{f 2}$ are the actual velocity in the main throat, sub-throat 1 , and sub-throat 2 (along the pathline's tangential direction), respectively, i.e., $v_{0}=\frac{d L_{f_{0}}}{d t}$, and so on; $L_{f 0}, L_{f 1}$, and $L_{f 2}$ are the pathline length in the main throat, subthroat 1 , and sub-throat 2 , respectively. According to continuity equation:

$\rho \frac{\pi}{4} d_{0}^{2} v_{f 0}=\rho \frac{\pi}{4} d_{1}^{2} v_{f 1}+\rho \frac{\pi}{4} d_{2}^{2} v_{f 2}$

The combined equation in Eq. (8) can be arranged as:

$$
\left\{\begin{array}{c}
y_{1}^{\prime}=\frac{\frac{G_{1}-B_{1} y_{1}}{I_{1}}+\frac{E_{1}}{F_{1} y_{2}-I_{1}} y_{2}-\frac{A_{1}-G_{1}}{F_{1} y_{2}-I_{1}}}{\frac{H_{1}}{F_{1} y_{2}-I_{1}}+\frac{H_{1}+C_{1} y_{1}}{I_{1}}} \\
y_{2}^{\prime}=\frac{G_{1}-B_{1} y_{1}}{I_{1}}-\frac{H_{1}+C_{1} y_{1}}{I_{1}} \frac{\frac{G_{1}-B_{1} y_{1}}{I_{1}}+\frac{E_{1}}{F_{1} y_{2}-I_{1}} y_{2}-\frac{A_{1}-G_{1}}{F_{1} y_{2}-I_{1}}}{\frac{H_{1}}{F_{1} y_{2}-I_{1}}+\frac{H_{1}+C_{1} y_{1}}{I_{1}}}
\end{array}\right.
$$

The imbibition distance is timed after the branch point. At $\mathrm{t}=0$, the imbibition length $y_{1}, y_{2}$ is 0 , i.e., the initial conditions are

$\left.y_{2}\right|_{t=0}=0,\left.\quad y_{1}\right|_{t=0}=0$

Equation (13) is a binary first-order non-linear ordinary differential equation, and its analytic solutions are difficult to obtain. Within an allowable error range, it may be solved numerically. This article employs variable-step fourth-fifth-order Runge-Kutta algorithm, which is an adaptive-step numerical analysis method for ordinary differential equations with a global truncation error of $(\Delta x)^{5}$. This problem can be solved by using a no stiff ordinary differential equation. On the basis of the abovementioned algorithms,

$$
\left\{\begin{array}{c}
P_{C 1}-P_{C 2}-L_{C 1} \cos \varphi_{1} \rho g-\frac{32 \mu L_{f 1} v_{f 1}}{d_{1}^{2}}=-L_{C 2} \cos \varphi_{2} \rho g-\frac{32 \mu L_{f 2} v_{f 2}}{d_{2}^{2}} \\
P_{C 1}-L_{C 0} \cos \varphi_{0} \rho g-L_{C 1} \cos \varphi_{1} \rho g-\frac{32 \mu L_{f 1} v_{f 1}}{d_{1}^{2}}=\frac{32 \mu L_{f 0} d_{1}^{2}}{d_{0}^{4}} v_{f 1}+\frac{32 \mu L_{f 0} d_{2}^{2}}{d_{0}^{4}} v_{f 2}
\end{array}\right.
$$

For straight throats, $L_{f 1}=L_{C 1}, L_{f 2}=L_{C 2}, L_{f 0}=L_{C 0}$. To clarify expressions, let us suppose that

$$
\left\{\begin{array}{l}
v_{f 1}=v_{C 1}=\frac{d L_{C 1}}{d t}=y_{1}^{\prime}=\frac{d y_{1}}{d t} \\
v_{f 2}=v_{C 2}=\frac{d L_{C 2}}{d t}=y_{2}^{\prime}=\frac{d y_{2}}{d t}
\end{array}\right.
$$

After Eq. (7) is integrated into Eq. (10), the following simplification is obtained:

$$
\left\{\begin{array}{c}
A_{1}-B_{1} y_{1}-C_{1} y_{1} y_{1}^{\prime}=E_{1} y_{2}+F_{1} y_{2} y_{2}^{\prime} \\
G_{1}-B_{1} y_{1}-C_{1} y_{1} y_{1}^{\prime}=H_{1} y_{1}^{\prime}+I_{1} y_{2}^{\prime}
\end{array}\right.
$$

where the constants $A_{1}=\frac{4 \sigma \cos \theta}{d_{1}}-\frac{4 \sigma \cos \theta}{d_{2}}, B_{1}=\rho g \cos \varphi_{1}, C_{1}=\frac{32 \mu}{d_{1}^{2}}$, $E_{1}=-\rho g \cos \varphi_{2}, \quad F_{1}=-\frac{32 \mu}{d_{2}^{2}}, \quad G_{1}=\frac{4 \sigma \cos \theta}{d_{1}}-L_{C 0} \rho g \cos \varphi_{0}$, $H_{1}=\frac{32 \mu L_{f 0} d_{1}^{2}}{d_{0}^{4}}$, and $I_{1}=\frac{32 \mu L_{f 0} d_{2}^{2}}{d_{0}^{4}}$.

The expression form of Eq. (12) shows that the time-dependent equation for the distance of the front of the imbibition in the two sub-throats is a binary first-order non-linear ordinary differential equation. Eq. (12) is sorted into an iterative normal equation using a one-degree term as the first-order term the initial conditions Eq. (14) are plugged into Eq. (13) by programming in MATLAB to find the numerical solutions of this equation. The results will be discussed in the next section.

For tortuous throats, fractal dimension is introduced as throat curvature, i.e., Eqs. (4) and (5). Thus,

$L_{f 1}=d_{1}{ }^{1-D_{T}} L_{C 1}^{D_{T}}, L_{f 2}=d_{2}{ }^{1-D_{T}} L_{C 2}^{D_{T}}$

The relation of actual velocity $v_{f}$ and linear velocity $v_{C}$ can be expressed as

$v_{f 1}=D_{T} L_{C 1}^{D_{T}-1} d_{1}^{1-D_{T}} v_{C 1}, v_{f 2}=D_{T} L_{C 2}^{D_{T}-1} d_{2}^{1-D_{T}} v_{C 2}$

To clarify expressions, suppose

$\left\{\begin{array}{l}v_{C 1}=\frac{d L_{C 1}}{d t}=y_{1}^{\prime}=\frac{d y_{1}}{d t} \\ v_{C 2}=\frac{d L_{C 2}}{d t}=y_{2}^{\prime}=\frac{d y_{2}}{d t}\end{array}\right.$

The equations for the movement laws of the front edge of spontaneous imbibition in sub-throats 1 and 2 (if tortuous) are drawn by integrating Eqs. (15) and (16) into Eq. (1), and then simplified as 
$\left\{\begin{array}{c}A_{2}-B_{2} y_{1}-C_{2} y_{1}^{2 D_{T}-1} y_{1}^{\prime}=E_{2} y_{2}+F_{2} y_{2}^{2 D_{T}-1} y_{2}^{\prime} \\ G_{2}-B_{2} y_{1}-C_{2} y_{1}^{2 D_{T}-1} y_{1}^{\prime}=H_{2} y_{1}^{D_{T}-1} y_{1}^{\prime}+I_{2} y_{2}^{D_{T}-1} y_{2}^{\prime}\end{array}\right.$

where the constants $A_{2}=\frac{4 \sigma \cos \theta}{d_{1}}-\frac{4 \sigma \cos \theta}{d_{2}}, \quad B_{2}=\rho g \cos \varphi_{1}$, $C_{2}=\frac{32 \mu D_{T}}{d_{1}^{2 D_{T}}}, E_{2}=-\rho g \cos \varphi_{2}, F_{2}=-\frac{32 \mu D_{T}}{d_{2}^{2 D_{T}}}, G_{2}=\frac{4 \sigma \cos \theta}{d_{1}}-L_{C 0} \rho g \cos \varphi_{0}$, $H_{2}=\frac{32 \mu L_{f 0} D_{T} d_{1}^{3-D_{T}}}{d_{0}^{4}}$, and $I_{2}=\frac{32 \mu L_{f 0} D_{T} d_{2}^{3-D_{T}}}{d_{0}^{4}}$. The above is still binary firstorder non-linear ordinary differential equations with the same processing method as the straight throat and are sorted into iterative normal equations of first-order term

$\left\{\begin{array}{c}y_{1}^{\prime}=\frac{G_{2}-B_{2} y_{1}-\left(A_{2}-B_{2} y_{1}-E_{2} y_{2}\right) /\left(F_{2} y_{2}^{D_{T}}\right)}{\left(C_{2}+H_{2} / y_{1}^{D_{T}}-I_{2} C /\left(F_{2} y_{2}^{D_{T}}\right)\right) y_{1}^{2 D_{T}-1}} \\ A_{2}-B_{2} y_{1}-E_{2} y_{2}-C_{2} \frac{G_{2}-B_{2} y_{1}-\left(A_{2}-B_{2} y_{1}-E_{2} y_{2}\right) /\left(F_{2} y_{2}^{D_{T}}\right)}{C_{2}+H_{2} / y_{1}^{D_{T}}-I_{2} C_{2} /\left(F_{2} y_{2}^{D_{T}}\right)} \\ F_{2} y_{2}^{2 D_{T}-1}\end{array}\right.$

The initial conditions are

$\left.y_{1}\right|_{t=0}=0,\left.y_{2}\right|_{t=0}=0$

Similarly, on the basis of fourth-fifth-order Runge-Kutta algorithm, $y_{1}$ and $y_{2}$ are numerically solved by programming in MATLAB ODE45 numerical solver. The results will be discussed in the next section.

If time-dependent imbibition mass is taken into account, then Eq. (21) will be used for solving and to determine how the length changes over time

$M(t)=\sum_{i} \rho \frac{\pi}{4} d_{i}^{2} L_{f i}(t)=\sum_{i} \rho \frac{\pi}{4} d_{i}^{3-D_{T}} L_{C i}^{D_{T}}(t)$

where $i=$ main_tube,sub_tube, which is the throat in which spontaneous imbibition is in progress at $t$. In the main tube of tree-like bifurcated throat, the mass flow rate of imbibition is the length of imbibition distance multiply to the cross section of tube and density of liquid; While $i=s u b_{-} t u b e$, the mass flow rate is the sum mass flow rate of two tubes.

\section{Results and discussions}

\subsection{Characteristics of typical imbibition curve of basic representative elementary structure}

In view of the above basic representative elementary structure model, the equations for the time-dependent imbibition distance were derived and represented by asymmetric tree-like sub-straight and tortuous throat structures. At $D_{T}=1$, the equation can be simplified as that for the straight throat. Equations (13) and (19) are programmed in MATLAB to calculate their numerical solutions. The time-dependence of total imbibition mass is calculated, as shown in Fig. 3. The imbibition curve in logarithmic coordnates are shown in Fig. 4.

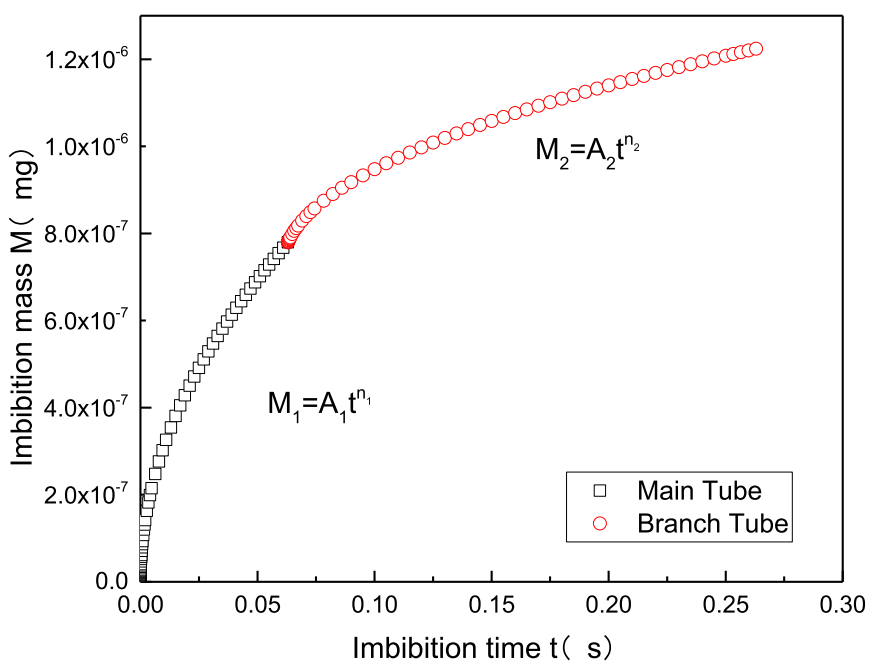

Fig. 3. Total imbibition mass curve with time (orthogonal coordinates),

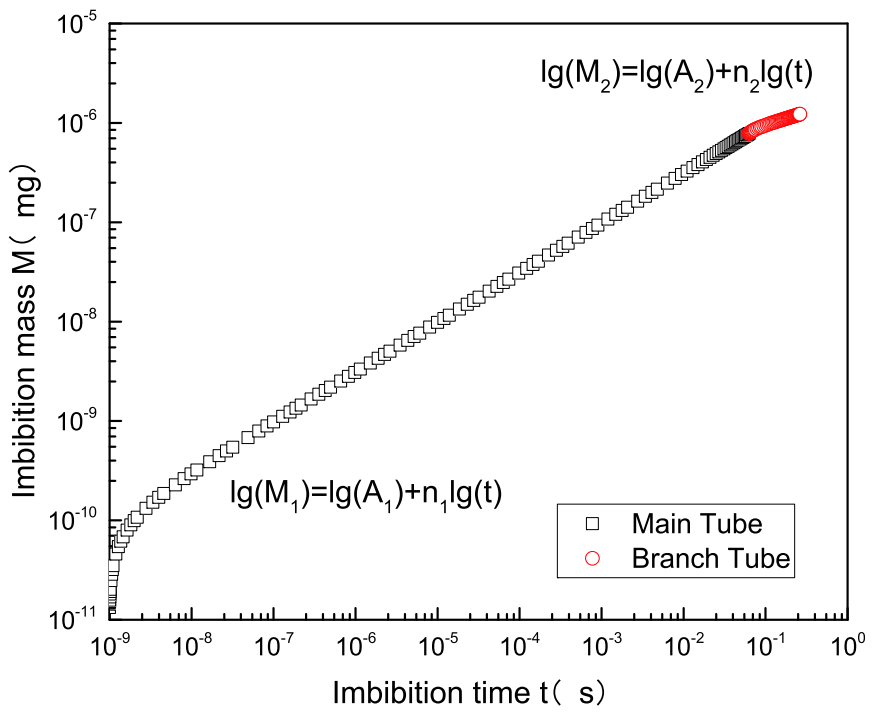

Fig. 4. Total imbibition mass curve with time (logarithmic coordinates).

$$
\text { (Constants : } \begin{aligned}
\sigma & =72.5 \mathrm{mN} / \mathrm{m}, \quad \rho=0.998 \mathrm{~g} / \mathrm{cm}^{3}, \mu \\
& =10^{-3} \text { Pa.s, } \quad \theta=30, g=9.8 \mathrm{~m} / \mathrm{s}^{2}, \quad d_{0} \\
& =1 \mu m, \quad d_{1}=d_{2}=0.5 \mu m, \quad D_{T}=1, \varphi_{0} \\
& =0^{0}, \varphi_{1}=\varphi_{2}=30^{0}, \quad L_{C 0}=L_{C 1}=L_{C 2} \\
& =1 \mathrm{~mm})
\end{aligned}
$$

Above is the basic rule of imbibition distance changing with time in a typical REV, which is derived from Eq. (21). Where the front of spontaneous imbibition does not reach the branch point, flow law is the tortuous single-capillary spontaneous imbibition, namely, in the main throat as illustrated; the spontaneous imbibition mass is increasing with time, while the slope is decreasing. Where it goes over the branch point, the fracturing fluid divides into two branches, and the imbibition proceeds, i.e., in the sub- 
throat as illustrated. If the main throat and the sub-throat are equal in length, the time to pass through the sub-throat is longer, and the slope drops gradually. This condition indicates that every REV absorbs water quickly at the beginning of spontaneous imbibition and slows down gradually later on.

The physical significance of these parameters was detailed in the preceding part. In logarithmic coordinates, changes in the porethroat structure can be observed, especially the throat forking that can affect spontaneous imbibition curves. Therefore, regarding throat forking as a factor that affects spontaneous imbibition rules makes sense in this article. Spontaneous imbibition rate A mainly relates to pore diameter, contact angle, viscosity, and surface tension. Imbibition index $n$ mainly relates to pore-throat tortuosity and pore size distribution. Thus, the imbibition rules for actual core pore-throat structure will be studied later in this article on the basis of REV spontaneous imbibition rules. A notable detail is that, given the limitations by the geometrical dimensions of basic REV (the main throat and the sub-throat is millimeter to micron-sized in length), only the imbibition rate at the initial stage of spontaneous imbibition may be represented in the analysis that uses REV as the object of study. At the end of spontaneous imbibition, the physical and chemical reactions between clay minerals and fracturing fluids are critical for spontaneous imbibition curves, and this model will result in obvious deviations.

\subsection{Influence factors for imbibition rate}

In this section, the spontaneous imbibition rate is calculated through numerical simulation against different porosity, reservoir wettability, viscosity, and liquid-gas surface tension conditions, and the influences of rocks and fracturing fluids on this factor are discussed separately.

(Except variable declarations in figures, calculating parameters include $d_{0}=1 \mu \mathrm{m}, d_{1}=d_{2}=0.5 \mu \mathrm{m}, d_{0} / d_{1}=2, \sigma=72.5 \mathrm{mN} / \mathrm{m}$, $\rho=0.998 \mathrm{~g} / \mathrm{cm}^{3}, \quad \mu=10^{-3}$ Pa.s, $\quad \theta=30, \quad g=0 \mathrm{~m} / \mathrm{s}^{2}, \quad \varphi_{0}=0^{0}$, $L_{C 0}=L_{C 1}=L_{C 2}=1 \mathrm{~mm}, D_{T}=1$ )

Fig. 5 shows the changes of imbibition rate A with porosity, reservoir wettability, fracturing fluid viscosity, and liquid-gas surface tension. In basic REV, porosity is expressed by matrix throat diameter. As one of the most important rock parameters, porosity reflects the size of rock pore space. Fig. 5(a) shows that porosity significantly affects imbibition rate, which increases significantly with matrix throat diameter. High-permeability sandstone reservoir has large porosity and consists mainly of macropores. A tight reservoir has small porosity and consists mainly of micro-nano pores. Consequently, a low-porosity tight reservoir has an obvious negative effect on initial imbibition rate.

Wettability is another important feature of shale gas reservoir, and the contact angle between the fracturing fluid and pore

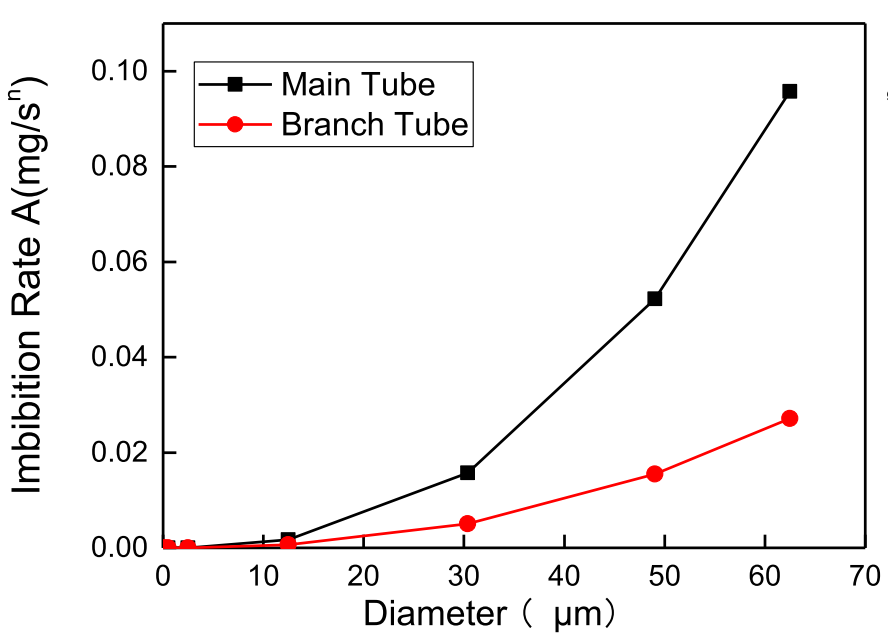

(a)

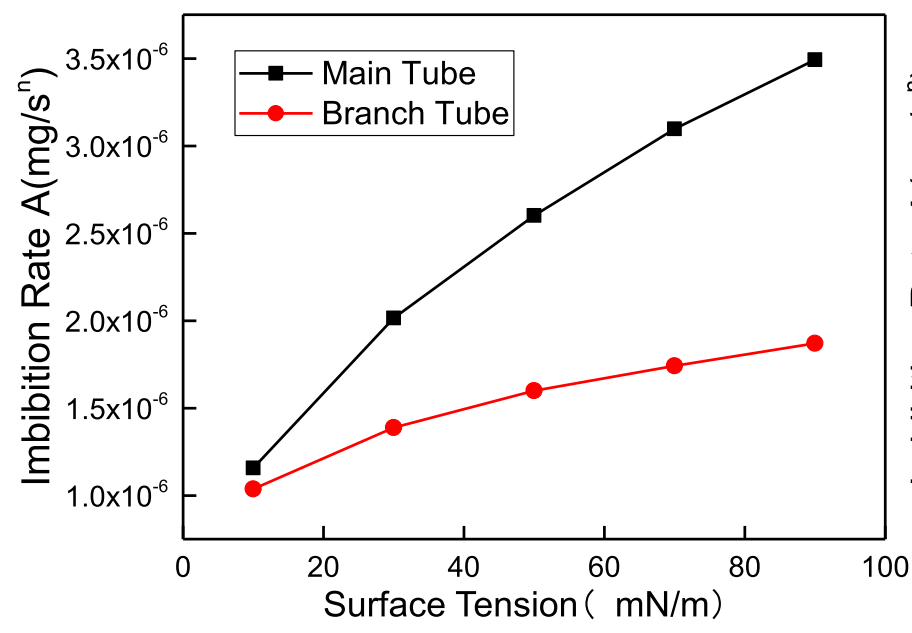

(c)

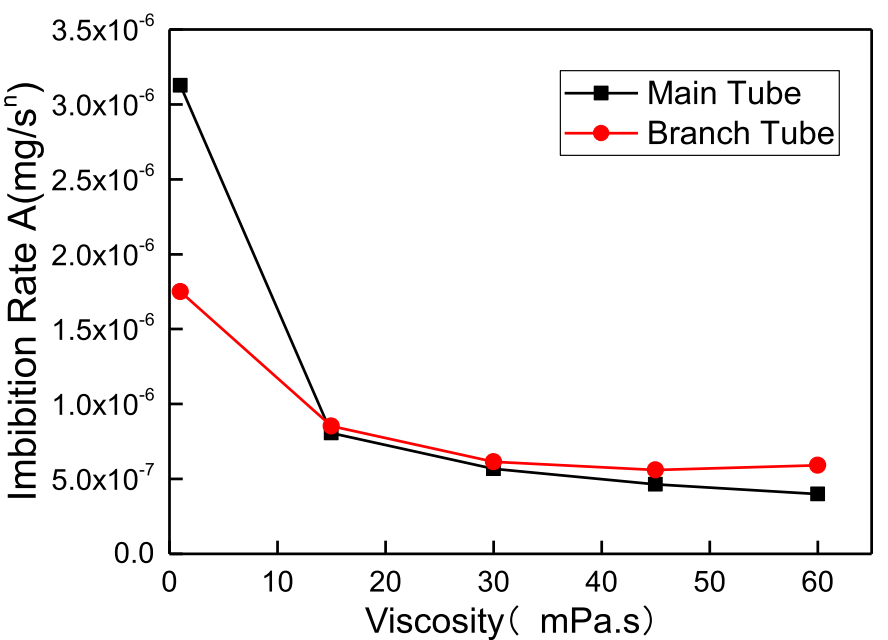

(b)

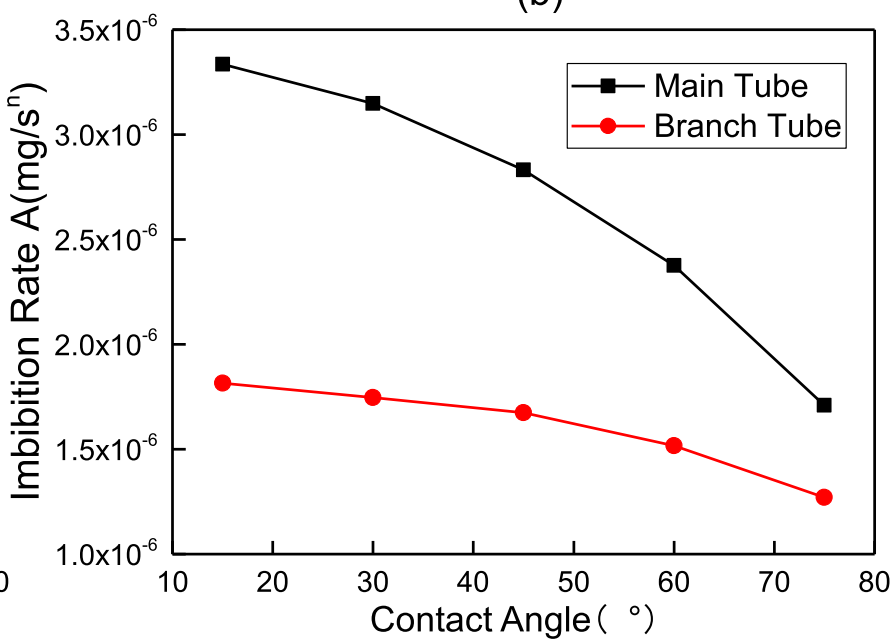

(d)

Fig. 5. Effect of various factors on imbibition rate. 


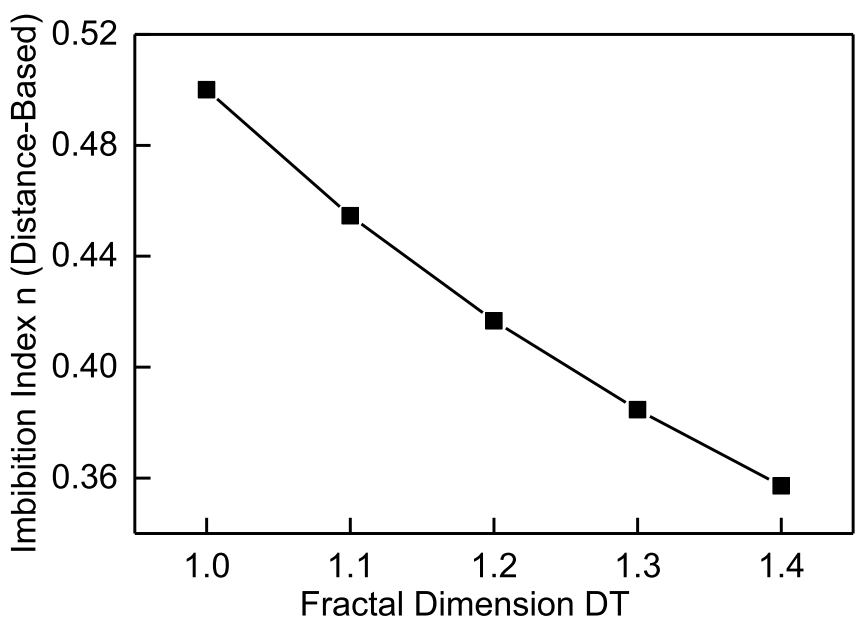

(a)

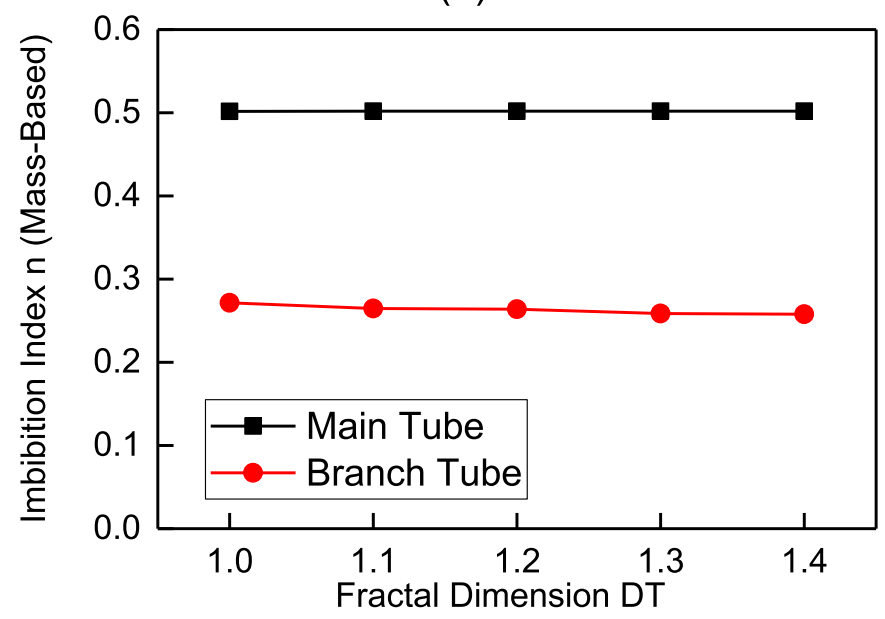

(b)

Fig. 6. Impact law of fractal dimension on SI index.

determines the capillary force on spontaneous imbibition. Fig. 5(b) shows that the matrix imbibition rate increases as the reservoir contact angle is reduced. This condition occurs because if fracturing fluid has good hydrophilicity, then capillary force and imbibition rate become stronger with the smaller reservoir contact angle. Therefore, in the fracturing process, the fracturing fluid imbibition rate can be improved by properly enhancing the hydrophilicity between fracturing fluid and rock to accelerate the removal of water blocks in fractures, increase the microscopic displacement effects, and enhance the initial recovery efficiency.

Viscosity is also an important index for fracturing fluids. Fig. 5(c) shows that imbibition rate drops with the increasing fracturing fluid viscosity. In addition, with low viscosity, changing the fracturing fluid density has even broader effects on spontaneous imbibition rate. Thus, high-viscosity fracturing fluid flow will cause high resistance, which is unfavorable for the spontaneous imbibition of fracturing fluids. With low-viscosity fracturing fluids, reducing viscosity could accelerate the spontaneous imbibition process dramatically.

Surface tension is another important index for fracturing fluids. Fig. 5(d) shows that spontaneous imbibition rate increases with the surface tension of fracturing fluid-natural gas in rock matrix throats. The increase of surface tension can induce the increase of capillary force. Thus, additional power needs to be provided for the spontaneous imbibition of fracturing fluids to increase the spontaneous imbibition rate.

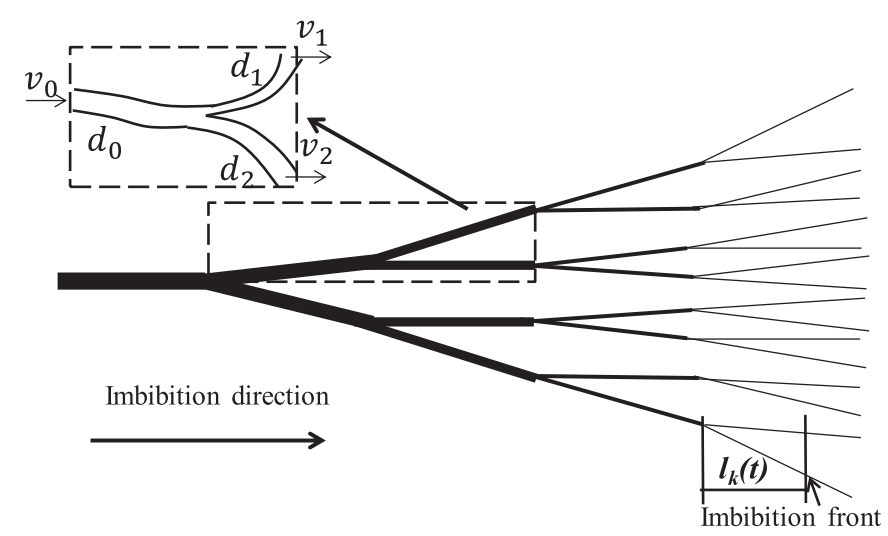

Fig. 7. Pore-throat spectrum with REV-characteristic complicated pore-throat structure.

\subsection{Influence factors for imbibition index}

In this section, throat tortuosity and pore size distribution on the imbibition index are calculated through a numerical simulation.

Fig. 6(b)shows that the mass-based imbibition index did not change significantly with fractal dimension; this finding is consistent with the equation derived by Cai et al., (2010a). As a result, the imbibition index is eliminated. In real cores, imbibition index relates to both throat tortuosity and pore-throat connectivity. However, this REV model neglected pore-throat connectivity. Thus, imbibition index is slightly affected by fractal dimension. If the ratio of imbibition mass to spontaneous imbibition distance is deemed constant, then the imbibition index based on imbibition distance decreases significantly as fractal dimension increases, as shown in Fig. 6(a). This result indicates that a great throat tortuosity corresponds to a high resistance and, in turn, a small imbibition index.

Pore size also has significant effects on rock imbibition characteristics. The microscopic pore structure of high-permeability sandstone is generally characterized by macro pores; the throat diameter mainly focuses on micron-sized pores, and it manifests as a simple, narrow peak on a throat distribution spectrum. This finding means that this rock has good connectivity and weak heterogeneity. However, tight reservoirs, especially shale, have micronano pores; the throat diameter varies from nanoscale to macro

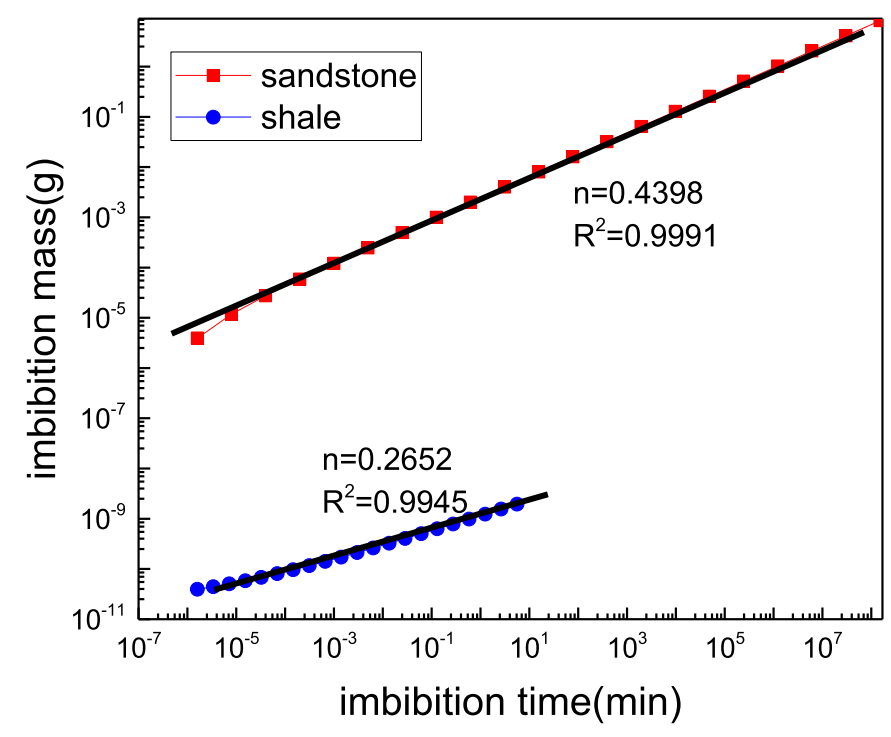

Fig. 8. Imbibition curve in simulated network (log-log coordinates). 
scale. On a pore size distribution spectrum, tight rock manifests as continuous low peaks, and the curve is wide. This finding means that the rock has bad connectivity and is heterogeneous. If fractures exist in shale, another peak will emerge. Briefly, a significant difference exists between maximum and minimum pores with strong heterogeneity in shale. Core pore structure distribution is complicated. In this paper, such a complicated spatial pore structure is equivalent to a network of pipes (Fig. 7), which consists of the REV represented by an asymmetric branch-like throat structure, and the diameters of pores in core distribution laws are similar to single

Table 1

Dimensions, porosity and permeability of various samples.

\begin{tabular}{lllll}
\hline Sample & Diameter $[\mathrm{mm}]$ & Length $[\mathrm{mm}]$ & Porosity [\%] & Permeability $[\mathrm{mD}]$ \\
\hline Shale1 & 25.01 & 17.56 & 5.50 & 0.0067 \\
Shale2 & 25.00 & 18.12 & 6.10 & 0.0082 \\
Sandstone1 & 24.99 & 15.19 & 11.20 & 0.1600 \\
Sandstone2 & 25.24 & 13.41 & 13.36 & 0.0389 \\
\hline
\end{tabular}

Table 2

Bulk mineralogy test result.

\begin{tabular}{|c|c|c|c|c|c|c|c|}
\hline Sample & Quartz & $\begin{array}{l}\text { Potassium } \\
\text { feldspar }\end{array}$ & $\begin{array}{l}\text { Plagioclase } \\
\text { feldspar }\end{array}$ & Calcite & Dolomite & Siderite Pyrite & $\begin{array}{l}\text { Total } \\
\text { clay }\end{array}$ \\
\hline shale & 24 & 22.5 & 2.6 & 6.6 & 2 & & 42.3 \\
\hline sandstone & 50.2 & 1.9 & 24.5 & & 2.3 & 3.2 & 20.7 \\
\hline
\end{tabular}
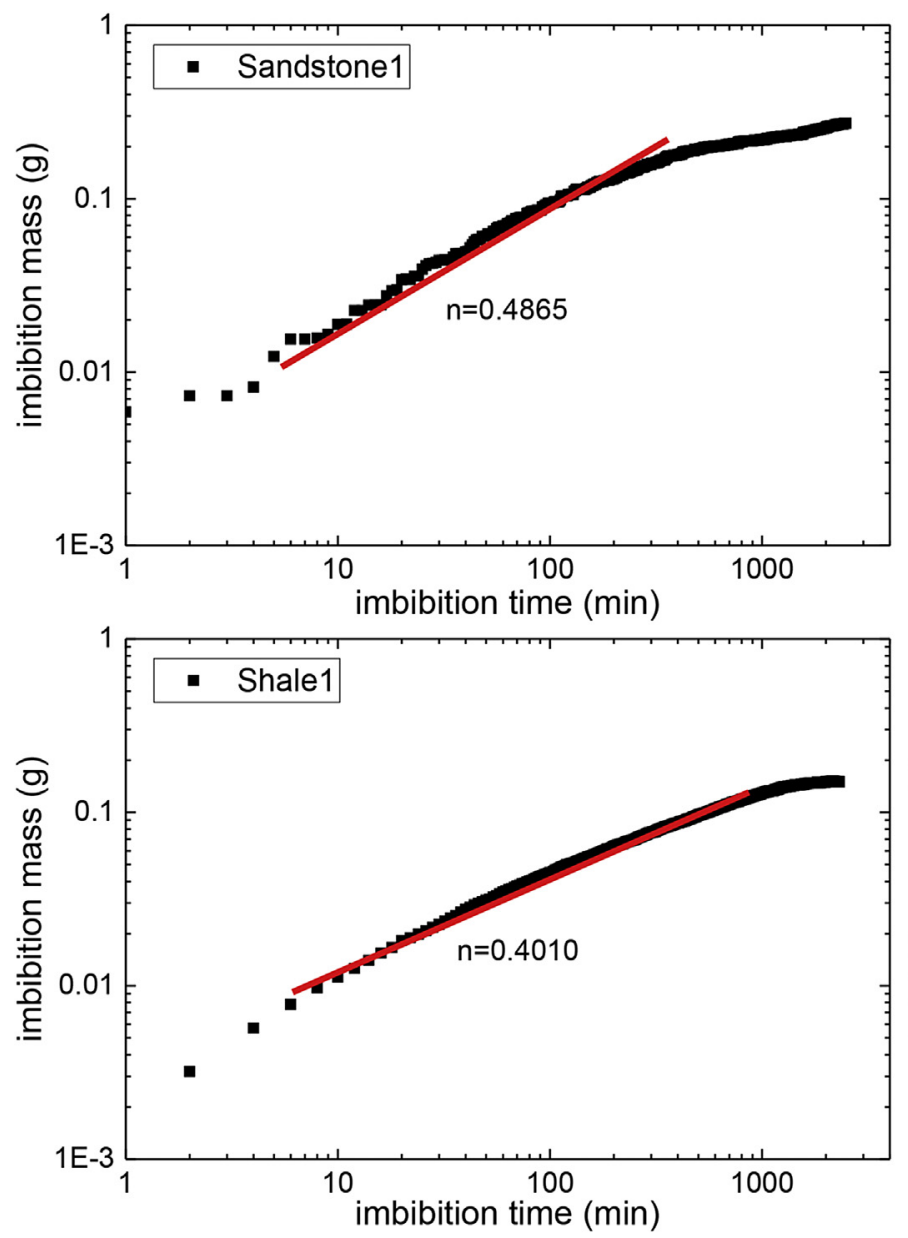

REV throat distribution laws.

In Fig. 7, Every imbibition unit is an asymmetric branch-like throat structure. Calculate equations and boundary conditions are discussed in the last section. To simulate the pore structure of the tight reservoir, $d_{0}=0.01 \mu \mathrm{m}, d_{1}=0.001 \mu \mathrm{m}$ and $d_{3}=0.001 \mu \mathrm{m}$ are adopted, and $d_{0}=d_{1}=d_{2}=1 \mu \mathrm{m}$ is used for high-permeability reservoir. Each Tube's length is 5 times than its diameter of main tube. Other parameters are set the same as Fig. 5. Fig. 8 is the calculated imbibition result.

Fig. 8 presents the simulated network imbibition curves for high-permeability and shale. This figure shows that the imbibition curves in log-log coordinates basically have linear regularity. As introduced in section 2, imbibition rate $A$ is intercept of curve in $\log$-log figure, which reflects the speed of imbibition mass at the initial stage of spontaneous imbibition. Imbibition rate in the highpermeability sandstone is significantly greater than that in shale. The imbibition index $n$ of sandstone is 0.4398 , while the imbibition index $\mathrm{n}$ of shale is 0.265 in our complicated pore-throat structure model. This finding means that pore size has significant influence on spontaneous imbibition characteristic. Larger pores gain higher imbibition rate and higher imbibition index.

To verification this summary, we also have taken some spontaneous imbibition experiments for sandstone and shale. We have tested series of sandstone and shale, the dimensions, porosity and permeability of various samples are listed in Table 1 . The shales are from Longmaxi formation of Sichuan Basin. The sandstones are from Chang-7 formation of Erdos Basin. And the bulk mineralogy
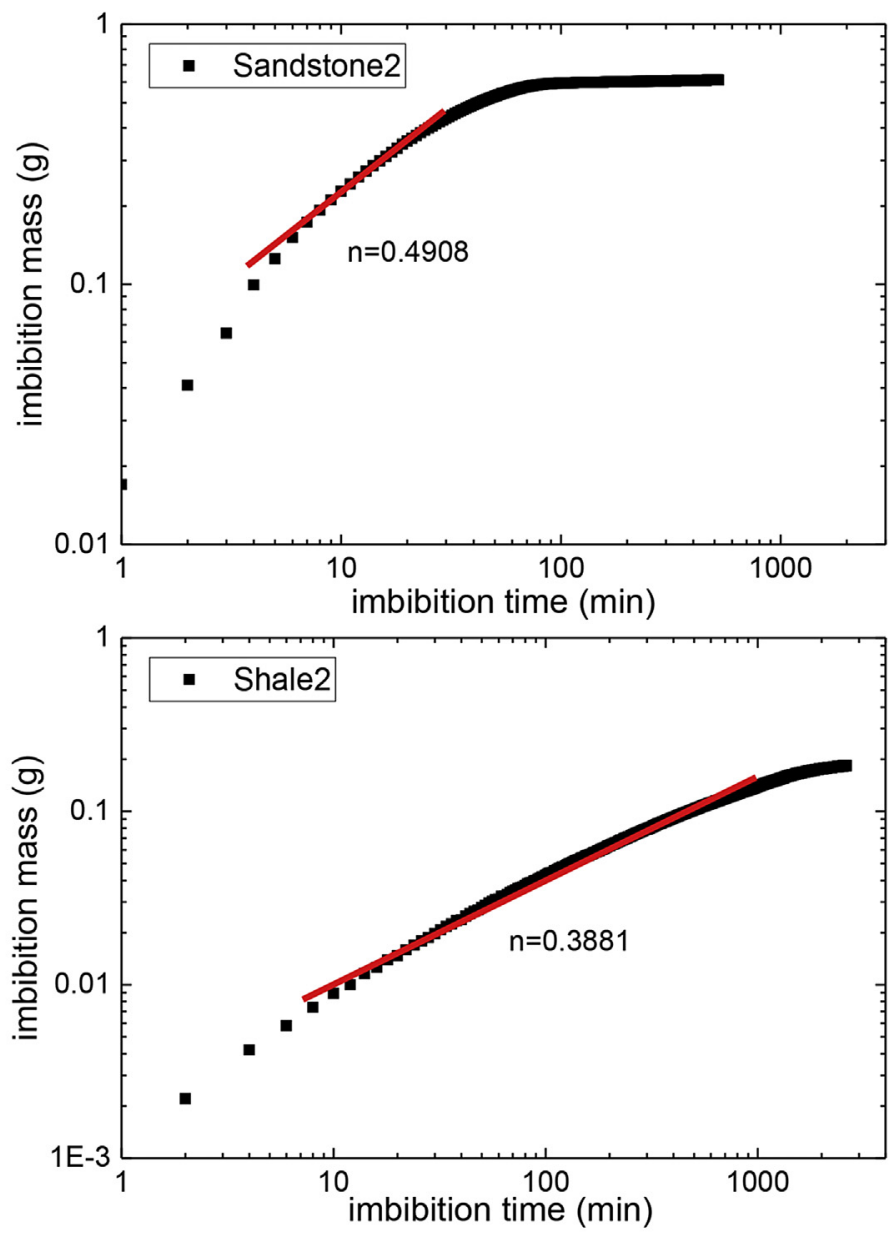

Fig. 9. Imbibition curve for the shale and sandstone samples (log-log coordinates). 
test result of samples are listed in Table 2.

The spontaneous imbibition tests are using an auto metering system. The imbibed water weight was measured on line by a balance (METTLER LE204E) connected to a computer. The experiments were performed at room pressure $(0.1 \mathrm{MPa})$ and temperature $\left(25^{\circ} \mathrm{C}\right)$. The results are shown in log-log coordinate (Fig. 9). The $\mathrm{n}$ values of the imbibition curves are totally different between sandstone and shales. The shales have a value lower than 0.5 .

To investigate the influence of pore size to the imbibition, the nuclear magnetic resonance (NMR) tests have been introduced. The fluid in large pores has longer T2 value because more nuclei are available to exhibit the NMR effect, and fluid in small pores has short T2 value. T2 relaxation time is in inverse proportion to specific surface of samples NMR technique has been widely used in rock analysis. The results are as shown in Fig. 10, which indicate that the sandstone's pore diameter is much higher than that of the shales.

For shale, spontaneous imbibition process is much slower than sand stones, The time spend reaching branching point in shale is hundred times than sand stones. The imbibition index $\mathrm{n}$ is a good parameter that reflects pore structure in tight reservoirs. In highpermeability reservoirs, the imbibition index $n$ is always approximately 0.5 whether the capillary is split or not. However, an important detail is that the imbibition index $\mathrm{n}$ reflects the pore structure in shale. If the pore space structure is simple, then $n$ is approximately 0.5 . However, if the pore space structure is complex,

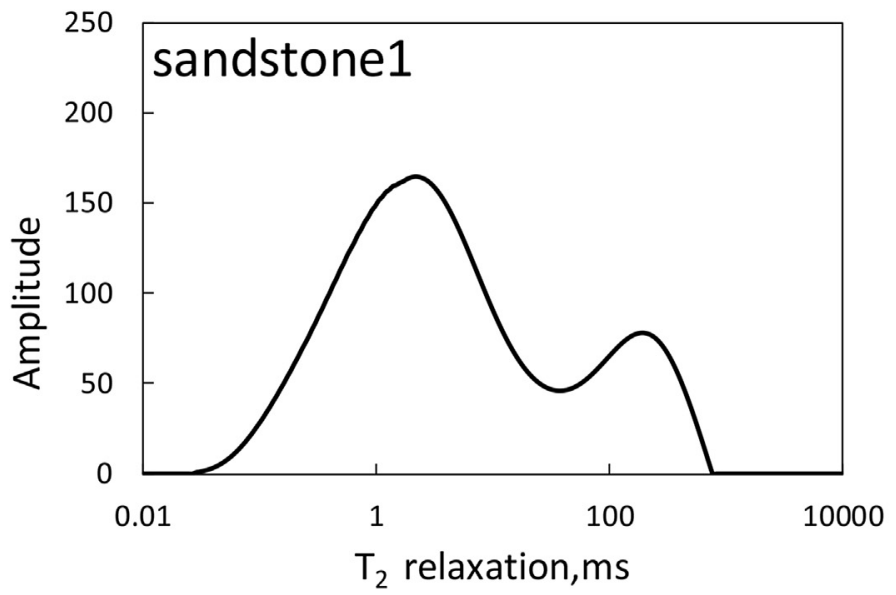

(a)

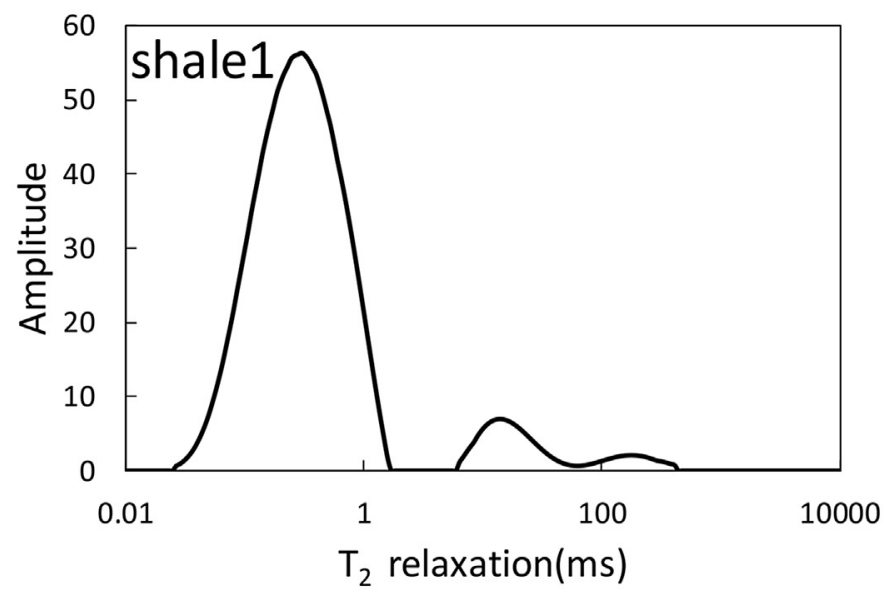

(c) like capillary forking, then $\mathrm{n}$ becomes small. Thus, for a shale core, if $n$ is small, then this core has a complex throat structure.

\section{Conclusions}

We began this paper by examining the spontaneous imbibition of fracturing fluids and studying factors that affect spontaneous imbibition in rocks. Pore space is equivalent to a pore network and consists of the REV represented by an asymmetric branch-like structure. A mathematical model is established and solved based on this asymmetric branch-like throat structure. The following findings were obtained:

The imbibition index and rate depends on both rock skeleton structure and fracturing fluid properties. To enhance efficiency in the initial stages of spontaneous imbibition, using low-viscosity fluids and even fresh water, enhancing hydrophilicity between fracturing fluid and rock are recommended for fracturing.

Imbibition rate and index are appropriate parameters for evaluating the spontaneous imbibition process. A sandstone has a much higher imbibition rate than a shale. In addition, both in models and experiments, imbibition index $\mathrm{n}$ is useful to describe imbibition properties of rocks. In unconventional rock samples like shale, $\mathrm{n}$ is less than 0.4 , while high-permeability sandstone, $\mathrm{n}$ approaches to 0.5 .

If the core sample shows weak spontaneous imbibition, then rapid flow back is required to prevent water blocking in the

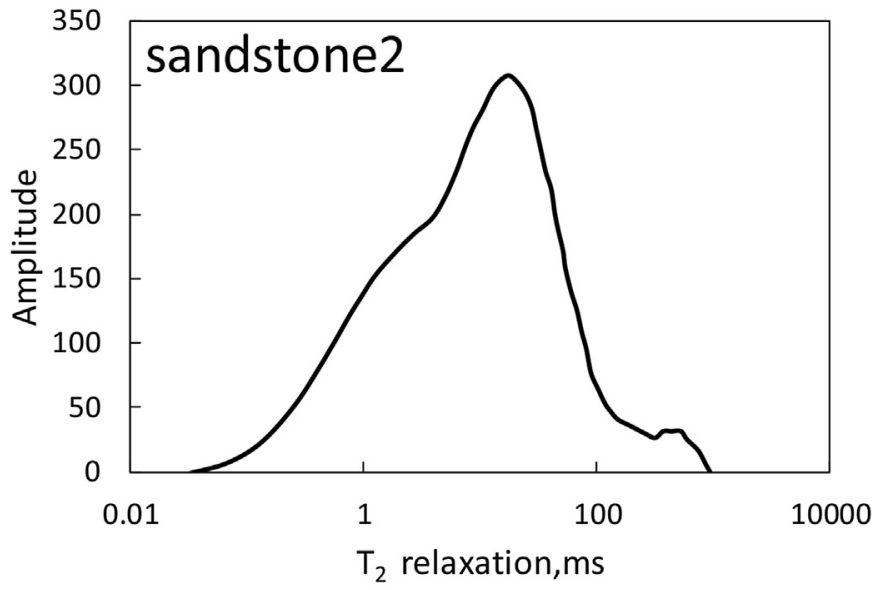

(b)

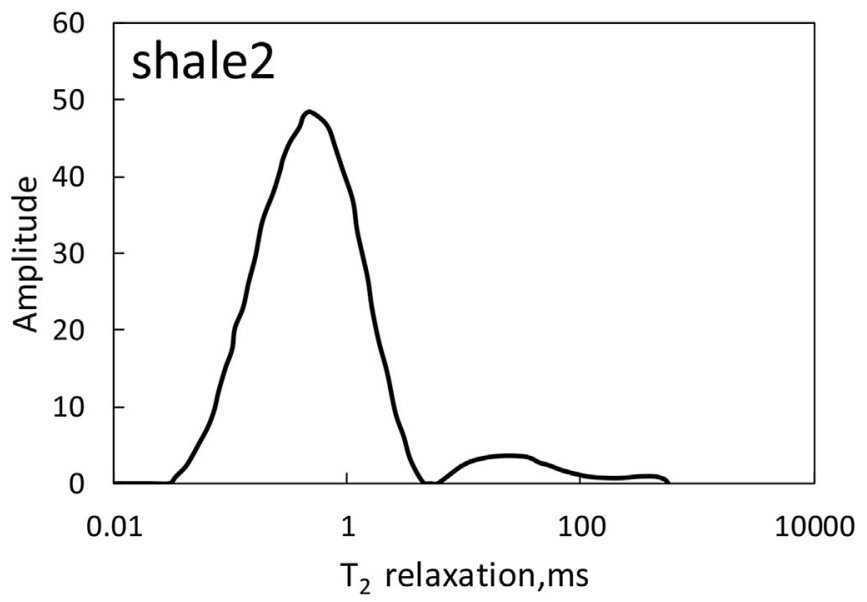

(d)

Fig. 10. Imbibition curve for the shale and sandstone samples (log-log coordinates). 
reservoir. However, if the core sample shows strong spontaneous imbibition effect, extending the soak-in time is recommended. This approach may enhance microscopic displacement and shale gas production in specific condition. While, some influence are not taken into consideration, like the non-Newton characteristics of fluid, low connectivity of matrix, compressibility of gas and matrix, the scale effect of shale media are not discussed in this model, which should be taken into consideration in future work.

\section{Acknowledgement}

This study was supported by the National Natural Science Foundation of China (U1562215, 51490652 and 51604287), the Science Foundation of China University of Petroleum, Beijing (2462015YQ1202), and the Sinopec scientific research project (P15026).

\section{References}

Ankur, R., Edmund, P., Dunne, W.M., et al., 2007. Fractal characterization of fracture networks: an improved box-counting technique. J. Geophys. Res. Atmosph. 112 (B12), 229-247.

Bertoncello, A., Wallace, J., Blyton, C., Honarpour, M., Kabir, C.S., 2014. Imbibition and water blockage in unconventional reservoirs: well management implications during flowback and early production. SPE Reserv. Eval. Eng. 17, 497-506.

Cai, J., Yu, B., 2011. A discussion of the effect of tortuosity on the capillary imbibition in porous media. Transp. Porous Media 89 (2), 251-263.

Cai, J., Yu, B., Mei, M.F., 2010a. Capillary rise in a single tortuous capillary. Chin. Phys. Lett. 27 (5), 054701.

Cai, J., Yu, B., Zou, M., Luo, L., 2010b. Fractal characterization of spontaneous cocurrent imbibition in porous media. Energy Fuel 24 (3), 1860-1867.

Cai, J., Hu, X., Standnes, D.C., et al., 2012. An analytical model for spontaneous imbibition in fractal porous media including gravity. Colloid Surf. A 414 (46), $228-233$.

Cai, J., Perfect, E., Cheng, C., et al., 2014. Generalized modeling of spontaneous imbibition based on Hagen-Poiseuille flow in tortuous capillaries with variably shaped apertures. Langmuir 30 (18), 5142-5151.

Cai, J., Luo, L., Ye, R., et al., 2015. Recent advances on fractal modeling of permeability for fibrous porous media. Fractals 23 (1), 1540006.

Clarkson, C.R., Jensen, J.L., Blasingame, T., 2011. Reservoir engineering for unconventional reservoirs: what do we have to consider. In: North American Unconventional Gas Conference and Exhibition (Soc. of Pet).

Engelhardt, W.V., Tunn, W.L.M., 1955. The flow of fluids through sandstones. Circ. III. State. Geol. Surv. 194, 1-17.

Handy, L.L., 1960. Determination of effective capillary pressures for porous media from imbibition data. Pet. Trans. AIME 219, 75-80.

Hu, Q., Ewing, R.P., Dultz, S., 2012. Low pore connectivity in natural rock. J. Contam. Hydrol. 76-83.
Katz, A.J., Thompson, A.H., 1985. Fractal sandstone pores: implications for conductivity and pore formation. Phys. Rev. Let. 54 (12), 1325-1328.

Krohn, C.E., 1988. Sandstone fraetal and Euelidean pore volume distributions. J.Geophys. Res. 93 (B4), 3286-3296.

Krohn, C.E., Thompson, A.H., 1986. Fractal sandstone pores: automated measurements using scanning-electron-microscope images. Phys. Rev. B 33 (9), 6366-6374

Li, K., Horne, R.N., 2001. Characterization of spontaneous water imbibition into gassaturated rocks. SPEJ 6 (4), 375-384.

Lucas, R., 1918. Rate of capillary ascension of liquids. Kolloid Z 23 (15), 15-22.

Luo, L., Yu, B., Cai, J., Mei, M., 2010. Symmetry is not always prefect. Int. J. Heat. Mass Transf. 53 (21), 5022-5024.

Makhanov, K., Habibi, A., Dehghaanpour, H., et al., 2014. Liquid uptake of gas shales: a workflow to estimate water loss during shut-in periods after fracturing operations. J. Uncon. Oil Gas. Res. 22-32.

Mandelbrot, B.B., 1982. The Fractal Geometry of Nature. W.H. Freeman, New York.

Moghanloo, R.G. Yuan, B. Ingrahama, N., et al., 2015. Applying macroscopic material balance to evaluate dynamic drainage volume and performance prediction of shale oil/gas wells. J. Nat. Gas. Sci. Eng. 27, 466-478.

Pia, G. 2016. Fluid flow in complex porous media: experimental data and IFU model predictions for water vapour permeability. J. Nat. Gas. Sci. Eng. 35, 283-290.

Pia, G., Casnedi, L., Sanna, U., 2016. Pore size distribution influence on suction properties of calcareous stones in cultural heritage: experimental data and model predictions. Adv. Mater. Sci. Eng. 2016.

Qasem, F.H., Nashawi, I.S., Gharbi, R., Mir, M.I., 2008. Recovery performance of partially fractured reservoirs by capillary imbibition. J. Pet. Sci. Eng. 60 (1), 39-50.

Rose, W., 2001. Modeling forced versus spontaneous capillary imbibition processes commonly occurring in porous sediments. J. Pet. Sci. Eng. 30 (3), 155-166.

Roychaudhuri, B., Tsotsis, T.T., Jessen, K., 2011. An experimental and numerical investigation of spontaneous imbibition in gas shales. In: SPE Annual Technical Conference and Exhibition (Soc. of Pet).

Sang, N., 2012. Study on Spontaneous Imbibition in Tree-like Branched Structure. Huazhong University of Science and Technology.

Schechter, D.S., Zhou, D., Orr, F.M., 1994. Low IFT drainage and imbibition. J. Pet. Sci. Eng. 11 (4), 283-300.

Shen, Y., Ge, H., Li, C., et al., 2016. Water imbibition of shale and its potential influence on shale gas recovery - a comparative study of marine and continental shale formations. J. Nat. Gas. Sci. Eng. 35, 1121-1128.

Washburn, E.W., 1921. The dynamics of capillary flow. Phys. Rev. 17 (3), 273-283.

Xu, P., Yu, B., Feng, Y., Zou, M., 2006. Permeability of the fractal disk-shaped branched network with tortuosity effect. Phys. Fluids 18 (7), 078103.

Yu, B., Cheng, P., 2002. A fractal permeability model for bi-dispersed porous media. J. Heat Mass Transf. 45 (14), 2983-2993.

Yuan, B., Su, Y., Moghanloo, R.G., 2015a. A New analytical multi-linear solution for gas flow toward fractured horizontal well with different fracture intensity. J. Nat. Gas. Sci. Eng. 23, 227-238.

Yuan, B., Wood, D.A., Yu, W., 2015b. Stimulation and hydraulic fracturing technology in natural gas reservoirs: theory and case study (2012-2015). J. Nat. Gas. Sci. Eng. 26, 1414-1421.

Zhang, X., Morrow, N.R., Ma, S., 1996. Experimental verification of a modified scaling group for spontaneous imbibition. SPERE 11 (4), 280-285. 